\title{
Impact of Compliance with an Enhanced Recovery After Surgery Program on the Outcomes Among Elderly Patients Undergoing Lumbar Fusion Surgery
}

This article was published in the following Dove Press journal: Clinical Interventions in Aging

\author{
Zhong-En $\mathrm{Li}^{1-3}$ \\ Shi-Bao Lu ${ }^{\mathrm{I}-3}$ \\ Chao Kong ${ }^{1-3}$ \\ Wen-Zhi Sun ${ }^{1-3}$ \\ Peng Wang ${ }^{1-3}$ \\ Si-Tao Zhang ${ }^{1,2}$ \\ 'Department of Orthopedics, Xuanwu \\ Hospital Capital Medical University, \\ Beijing, People's Republic of China; \\ ${ }^{2}$ National Clinical Research Center for \\ Geriatric Diseases, Beijing, People's \\ Republic of China; ${ }^{3}$ Capital Medical \\ University, Beijing, People's Republic of \\ China
}

Background and Aim: The benefits of the enhanced recovery after surgery (ERAS) program to elderly patients have not been evaluated in lumbar fusion surgery. Compliance with the ERAS program is associated with prognosis. There is currently no adequate assessment about the importance of the individual components of ERAS program in lumbar fusion surgery. The aim of the study was to analyze the effect of compliance with our ERAS program and the relative importance of the individual ERAS program components among elderly patients undergoing lumbar fusion surgery.

Methods: A retrospective case-review study was conducted from March 2018 to March 2020. The ERAS program for lumbar fusion surgery at our department was implemented. Overall compliance was found to be $92.9 \%$, and this was used as a cutoff for dividing patients into higher compliance and lower compliance groups. Patient characteristics and clinical outcomes were compared between groups.

Results: The overall compliance rate was $92.9 \%$, distributing 91 patients into the higher compliance group and 169 patients into the lower compliance group. Patients with higher compliance were younger $(\mathrm{p}=0.045)$. The length of stay (LOS) of patients with higher compliance was significantly shorter than that of patients with lower compliance. Patients with higher compliance had significantly fewer complications $(\mathrm{p}=0.031)$. A multivariate analysis showed that surgical time $(p=0.029)$, lower compliance $(p=0.034)$, and early oral feeding $(p=0.026)$ were predictors of any postoperative complications. On multivariate analysis, the following items remained correlated with prolonged $\operatorname{LOS}$ ( $\operatorname{LOS} \geq 12$ days): older age $(\mathrm{p}=0.010)$, lower compliance $(p<0.0001)$, early ambulation $(p=0.018)$, and stick to discharge criteria $(p=0.040)$.

Conclusion: Low compliance with ERAS program among elderly patients undergoing lumbar fusion surgery was associated with a higher incidence of complications and prolonged LOS. The failure of early oral feeding was associated with increased complications, and the failure of early ambulation or sticking to discharge criteria was significantly more influential on prolonged LOS.

Keywords: compliance, elderly, enhanced recovery after surgery; ERAS, lumbar fusion surgery

\section{Introduction}

Enhanced recovery after surgery (ERAS) program is an evidence-based method that is focused on improving postoperative recovery. ${ }^{1,2}$ It was originally developed based on a study in elderly patients undergoing colon surgery, and has since been implemented in several other surgical areas. More recently the ERAS program was proven to be feasible and efficacious in reducing complications and length of stay (LOS) after lumbar fusion surgery. ${ }^{3,4}$ 
The number of elderly patients who need to undergo lumbar surgery has significantly increased. However, the postoperative complications are more prevalent in this patient group, and have long been the main causes of prolonged LOS and increased care costs. ${ }^{5}$ Enhancing the perioperative management and reducing peri-operative stress can be a cost-effective way to improve the safety in elderly patients. ${ }^{6}$

An ERAS program encompasses many elements from initial patient referral through discharge. The overall benefits can be attributed to the aggregation of marginal gains of each components. ${ }^{7}$ From the very beginning the ERAS program was designed with an emphasis on elderly patients as the target group. ${ }^{1}$ The application of the ERAS program can minimize the stress responses to support recovery, which is particularly important for the vulnerable elderly patients with co-morbidities. ${ }^{8}$ Although benefits of the ERAS program to elderly patients have been reported in several types of surgeries, similar evaluation has not been extended to lumbar fusion surgery.

One of the confounding factors for accurate assessment of the ERAS program in elderly patients is compliance with the care protocols. It has been reported that compliance with the ERAS program is strongly associated with prognosis. ${ }^{6,9}$ While strict compliance with ERAS protocols is more difficult for all elderly patients in general, it might be particularly challenging for those undergoing lumber fusion surgery. The requirement for active participation in the program may be considered as too aggressive by this patient group. ${ }^{10}$ Therefore further studies are required to assess the compliance with ERAS program in this particular population. ${ }^{10}$

The importance of the individual components of ERAS program in colorectal surgery had been reported that the minimally invasive surgical technique and avoidance of fluid overload was associated with the prognosis. ${ }^{11,12}$ Although, there is currently no adequate assessment about the different importance of singular elements of ERAS program in lumbar fusion surgery.

The aim of the present study was to analyze the effect of compliance with our ERAS program and the relative importance of the individual ERAS program components on postoperative complications and LOS among elderly patients undergoing lumbar fusion surgery.
Table I Components of Our ERAS Program

\begin{tabular}{|c|c|}
\hline & ERAS Program \\
\hline \multicolumn{2}{|l|}{ Pre-operative } \\
\hline Education & $\begin{array}{l}\text { Including the purpose, workflows and benefits of } \\
\text { ERAS program, anticipated postoperative pain } \\
\text { and expectations and risks of surgery, through } \\
\text { verbal and handouts }\end{array}$ \\
\hline Nutritional counselling & $\begin{array}{l}\text { Nutrition screening during the perioperative } \\
\text { period, including nutrition screening tools } \\
\text { and laboratory indicators. Dietitians provide } \\
\text { personalized diet guidance and nutritional } \\
\text { supplement to patients in need }\end{array}$ \\
\hline Fasting & $\begin{array}{l}\text { Clear fluids including carbohydrate drink } \\
\text { allowed up to } 2 \text { hours before surgery }\end{array}$ \\
\hline $\begin{array}{l}\text { Antithrombotic } \\
\text { prophylaxis }\end{array}$ & $\begin{array}{l}\text { Active/passive limb movement and } \\
\text { antithrombotic stockings }\end{array}$ \\
\hline $\begin{array}{l}\text { Antimicrobial } \\
\text { prophylaxis }\end{array}$ & Antibiotic prophylaxis within I hour of incision \\
\hline \multicolumn{2}{|l|}{ Intra-operative } \\
\hline Tranexamic acid & Used routinely \\
\hline $\begin{array}{l}\text { Standard anesthetic } \\
\text { protocol }\end{array}$ & $\begin{array}{l}\text { Multimodal analgesia; TIVA-based anesthetic } \\
\text { technique with propofol, lidocaine, ketamine, } \\
\text { ketorolac, antiemetics and with up to } 0.5 \% \text { MAC } \\
\text { inhaled anesthetics, avoid } \mathrm{N}_{2} \mathrm{O} \text {; depth of } \\
\text { anesthesia monitoring }\end{array}$ \\
\hline $\begin{array}{l}\text { Avoidance of salt and } \\
\text { water overload }\end{array}$ & Goal-directed euvolemia \\
\hline $\begin{array}{l}\text { Maintenance of } \\
\text { normothermia }\end{array}$ & Keeping core temperature at $36-37^{\circ} \mathrm{C}$ \\
\hline $\begin{array}{l}\text { Local infiltration } \\
\text { analgesia }\end{array}$ & Used routinely \\
\hline \multicolumn{2}{|l|}{ Post-operative } \\
\hline Early ambulation & $\begin{array}{l}\text { In-bed mobilization within } 4 \text { hours after surgery. } \\
\text { Encourage ambulation after } 4 \text { hours, Early } \\
\text { treatment with physical therapists }\end{array}$ \\
\hline $\begin{array}{l}\text { Early removal of } \\
\text { bladder catheter }\end{array}$ & $\begin{array}{l}\text { Remove the bladder catheter when returning } \\
\text { to the ward }\end{array}$ \\
\hline Early oral feeding & $\begin{array}{l}\text { Oral feeding at will after recovery from } \\
\text { anesthesia }\end{array}$ \\
\hline $\begin{array}{l}\text { Postoperative } \\
\text { multimodal analgesia }\end{array}$ & $\begin{array}{l}\text { Adequately controlled pain; visual analog scales } \\
<4 \text { : no analgesia or oral minimal dose of } \\
\text { nonopioid; visual analog scales: } 4-6 \text { : oral or } \\
\text { intravenous nonopioid visual analog scales } \geq 7 \text { : } \\
\text { opioid }\end{array}$ \\
\hline
\end{tabular}

(Continued) 
Table I (Continued).

\begin{tabular}{|l|l|}
\hline & ERAS Program \\
\hline Stick to Discharge & $\begin{array}{l}\text { Discharge Criteria: No clinical complications; } \\
\text { visual analog scales <3 with oral analgesics; } \\
\text { Independent ambulation or ambulation with } \\
\text { minimal assistance; adequate nutrition intake; } \\
\text { absence of fever in the last } 48 \text { hours }\end{array}$ \\
\hline
\end{tabular}

Abbreviations: TIVA, total intravenous anesthesia; MAC, minimum alveolar concentration.

\section{Methods Study Design}

A retrospective case-review study was conducted in the department of orthopedics, Xuanwu Hospital Capital Medical University, also known as National Clinical Research Center for Geriatric Diseases, from March 2018 to March 2020. The ERAS program for posterior lumbar fusion surgery at our department was implemented in January 2018. For the reliability of the data, we started to record the data two months after the implementation of ERAS. Elderly was defined in our study as aged older than 65 years. The inclusion criteria included patients older than 65 years who underwent open posterior lumbar fusion surgery with pedicle screw fixation. The main diagnosis was lumbar stenosis with instability, with some accompanied with scoliosis or spondylolisthesis. Exclusion criteria included the following: 1) loss of follow up; 2) circumferential fusion (a combination of anterior and posterior surgery); 3) a history of previous lumbar spine surgery; and 4) emergency operations. All patients underwent a standard midline posterior lumbar decompression and interbody fusion was preferred in the decompressed levels. All surgeries were performed by the same surgeon and the ERAS program was performed by the same group of trained anesthesiologists, surgeons and nurses. The ERAS program has been described in a previous publication by our group. ${ }^{13}$ The 15 components of our ERAS program are shown in Table 1. Compliance was recorded as the number of achieved items by dedicated stuff who did not participate in the study and the compliance rate was defined as the number of program items observed divided by the total number of items. Overall compliance was found to be $92.9 \%$, and this was used as

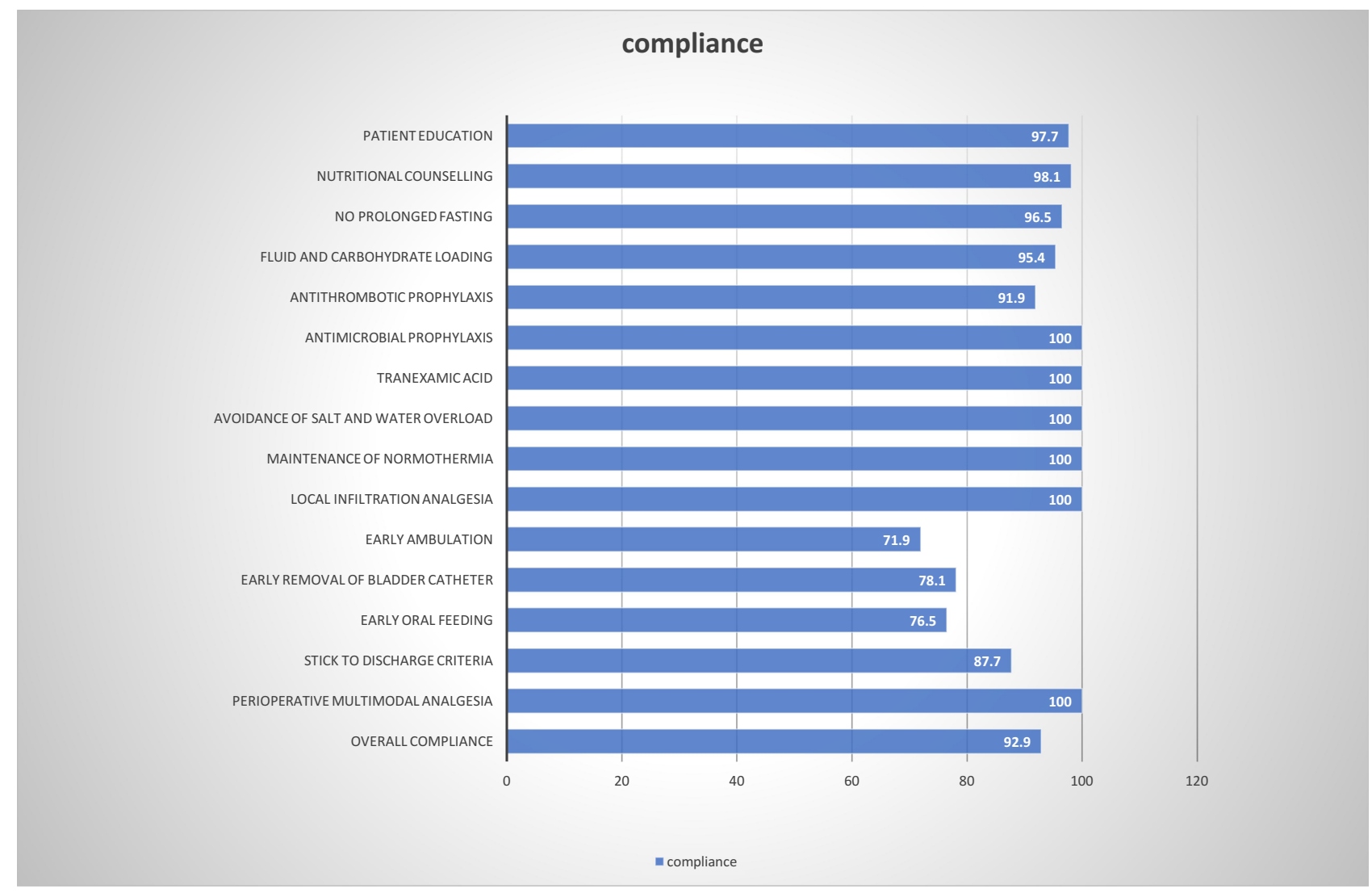

Figure I Compliance rate to individual elements of the ERAS program. 
a cutoff for dividing patients into higher compliance and lower compliance groups. During the study period, there were no major changes to the ERAS program at our department.

This study was approved by the institutional review board following the declaration of Helsinki principles in Xuanwu Hospital Capital Medical University (No. 2018008). All patients provided informed consent to the work.

\section{Data Collection}

Patient characteristics including age, gender, BMI, smoking status, comorbidities, American Society of Anesthesiologists (ASA) classification, preoperative Oswestry Disability Index (ODI), Visual Analogue Scale (VAS) of back, VAS of leg, surgical level, surgical time and estimated blood loss were collected from all patients. We also recorded the clinical outcomes, including LOS, complications and 30-day readmission. LOS was defined as the time from admission to discharge recorded in the medical record. And we defined prolonged LOS as a LOS $\geq 12$ days. Complications referred to all postoperative complications that occurred within 30 days after surgery and were determined by outpatient or follow-up doctors.

\section{Statistical Analysis}

Statistical analysis was performed using SPSS version 16.0 for Windows (SPSS Inc., Chicago, IL, USA). Continuous variables were expressed as mean value \pm standard deviation (Mean $\pm \mathrm{SD}$ ). Differences in mean values were tested with one-way analysis of variance and Student's $t$-test for normal data. Statistical analysis for categorical variable was performed by the Chi-square test. Univariate and multivariate logistic regression analyses for any complications and prolonged LOS were used to identify the influence of selected factors on clinical outcomes. $\mathrm{P}$ values $<0.05$ were considered statistically significant.

\section{Results}

The compliance rates of ERAS items are shown in Figure 1. In general, the pre-operative and intra-operative ERAS items achieved significant compliance, whereas compliance of the postoperative ERAS items was relatively low: early ambulation (71.9\%), early removal of bladder catheter (78.1\%), early oral feeding (76.5\%), stick to discharge criteria (87.7\%). The overall compliance rate was $92.9 \%$, distributing 91 patients into the higher compliance group $(>92.9 \%)$ and 169 patients into the lower compliance group $(\leq 92.9 \%)$.
Table 2 Patients' Characteristics

\begin{tabular}{|c|c|c|c|}
\hline & $\begin{array}{l}\text { Higher } \\
\text { Compliance } \\
(>92.9)\end{array}$ & $\begin{array}{l}\text { Lower } \\
\text { Compliance } \\
(\leq 92.9)\end{array}$ & $P$ value \\
\hline Patients (n) & 91 & 169 & \\
\hline Age, mean $\pm S D$ & $69.6 \pm 4.4$ & $73.3 \pm 7.1$ & 0.045 \\
\hline Gender & & & 0.834 \\
\hline Male & 40 & 72 & \\
\hline Females & 51 & 97 & \\
\hline BMI & $25.1 \pm 3.76$ & $26.2 \pm 4.14$ & 0.569 \\
\hline Smoker & 9 & 14 & 0.664 \\
\hline \multicolumn{4}{|l|}{ Comorbidities } \\
\hline Hypertension & 48 & 79 & 0.356 \\
\hline Diabetes & 36 & 55 & 0.258 \\
\hline $\begin{array}{l}\text { Ischemic heart } \\
\text { disease }\end{array}$ & 12 & 15 & 0.277 \\
\hline Arrhythmias & 5 & 5 & 0.310 \\
\hline Gastrointestinal & 4 & 8 & 0.901 \\
\hline Chronic lung disease & 5 & 6 & 0.458 \\
\hline Parkinson disease & 2 & 4 & 0.931 \\
\hline Depression & 6 & 7 & 0.387 \\
\hline ASA classification & & & 0.936 \\
\hline 1 & 2 & 5 & \\
\hline 2 & 53 & 98 & \\
\hline 3 & 36 & 66 & \\
\hline Preoperative ODI (\%) & $59.6 \pm 20.3$ & $64.2 \pm 15.1$ & 0.177 \\
\hline Preoperative VAS back & $6.6 \pm 1.9$ & $6.9 \pm 2.1$ & 0.644 \\
\hline Preoperative VAS leg & $7.78 \pm 2.0$ & $7.60 \pm 2.3$ & 0.738 \\
\hline Surgical level & & & 0.062 \\
\hline I-2levels & 65 & 101 & \\
\hline$\geq 3$ levels & 26 & 68 & \\
\hline Surgical time, min & $169.7 \pm 40.2$ & $180.4 \pm 52.6$ & 0.329 \\
\hline $\begin{array}{l}\text { Estimated blood } \\
\text { loss, mL }\end{array}$ & $322.5 \pm 276.9$ & $299.1 \pm 223.4$ & 0.138 \\
\hline
\end{tabular}

Abbreviations: ASA, American Society of Anesthesiologists; ODI, Oswestry Disability Index; VAS, Visual Analogue Scale.

There were no statistical differences in gender $(\mathrm{p}=0.834)$, BMI $(\mathrm{p}=0.569)$, smoker $(\mathrm{p}=0.664)$, any comorbidities, ASA classification ( $\mathrm{p}=0.936)$, preoperative ODI $(\mathrm{p}=0.177)$, preoperative VAS back $(\mathrm{p}=0.644)$, preoperative VAS leg $(\mathrm{p}=0.738)$, surgical level $(\mathrm{p}=0.062)$, surgical time $(\mathrm{p}=0.329)$ and estimated blood loss $(\mathrm{p}=0.138)$ between the two groups of patients. Notably, patients with higher compliance were younger (69.6 \pm 4.4 vs. $73.3 \pm 7.1$ years, $\mathrm{p}=0.045$ ). (Table 2).

The LOS of patients with higher compliance $(11.8 \pm 4.5$ days) was significantly shorter than that of patients with lower compliance (14.6 \pm 6.1 days). Patients with higher 
Table 3 Logistic Regression for Any Complications

\begin{tabular}{|c|c|c|c|c|}
\hline \multirow[t]{2}{*}{ Characteristics } & \multicolumn{2}{|c|}{ Univariable Analysis } & \multicolumn{2}{|c|}{ Multivariable Analysis } \\
\hline & OR $(95 \% \mathrm{Cl})$ & p value & OR $(95 \% \mathrm{Cl})$ & p value \\
\hline Age & $1.10(0.93-1.22)$ & 0.248 & & \\
\hline Gender (Female) & $0.83(0.42-1.62)$ & 0.576 & & \\
\hline BMI & $0.91(0.88-1.25)$ & 0.157 & & \\
\hline Surgical level $\geq 3$ & $0.84(0.42-1.68)$ & 0.621 & & \\
\hline$A S A \geq 3$ & $0.98(0.49-1.95)$ & 0.957 & & \\
\hline Smoker & $0.66(0.23-1.89)$ & 0.440 & & \\
\hline Preoperative ODI (\%) & $0.82(0.77-1.06)$ & 0.127 & & \\
\hline Preoperative VAS back & $0.98(0.88-1.14)$ & 0.861 & & \\
\hline Preoperative VAS leg & $0.89(0.74-1.30)$ & 0.363 & & \\
\hline Surgical time & $1.96(1.03-3.32)$ & 0.023 & $1.89(1.02-3.13)$ & 0.029 \\
\hline Estimated blood loss & $1.03(0.74-2.76)$ & 0.694 & & \\
\hline Lower compliance & $2.47(1.09-5.62)$ & 0.031 & $2.54(1.17-5.78)$ & 0.034 \\
\hline Patient education & $0.91(0.10-7.98)$ & 0.930 & & \\
\hline Nutritional counselling & $0.72(0.08-6.63)$ & 0.774 & & \\
\hline No prolonged fasting & $0.62(0.13-3.12)$ & 0.566 & & \\
\hline Fluid and carbohydrate loading & $0.91(0.19-4.29)$ & 0.900 & & \\
\hline Antithrombotic prophylaxis & $1.10(0.31-3.92)$ & 0.884 & & \\
\hline Antimicrobial prophylaxis & NA & NA & & \\
\hline Tranexamic acid & NA & NA & & \\
\hline Avoidance of salt and water overload & NA & NA & & \\
\hline Maintenance of normothermia & NA & NA & & \\
\hline Local infiltration analgesia & NA & NA & & \\
\hline Early ambulation & $0.90(0.43-1.87)$ & 0.769 & & \\
\hline Early removal of bladder catheter & $0.60(0.28-1.27)$ & 0.183 & & \\
\hline Early oral feeding & $0.42(0.19-0.92)$ & 0.030 & $0.39(0.17-0.91)$ & 0.026 \\
\hline Stick to discharge criteria & $0.98(0.35-2.72)$ & 0.968 & & \\
\hline Perioperative multimodal analgesia & NA & NA & & \\
\hline
\end{tabular}

Abbreviations: ASA, American Society of Anesthesiologists; ODI, Oswestry Disability Index; VAS, Visual Analogue Scale.

compliance had significantly fewer complications $(p=0.031)$. The details of complications are shown in Table 3. 30-day readmission was not impacted by the level of compliance $(p=0.298)$. Two patients of lower compliance group were readmitted within 30 days of discharge because of surgical site infection (SSI). During our follow-up, there was no occurrence of patient re-operation or death (Table 4).

A multivariate analysis showed that surgical time (OR 1.89, 95\% CI 1.02-3.13, $\mathrm{p}=0.029)$, lower compliance (OR $2.54,95 \%$ CI $1.17-5.78, \mathrm{p}=0.034)$, early oral feeding (OR $0.39,95 \%$ CI $0.17-0.91, \mathrm{p}=0.026$ ) were predictors of any postoperative complications (Table 3).

On multivariate analysis, the following items remained correlated with prolonged LOS (LOS $\geq 12$ days): older age (OR 1.45, 95\% CI 1.02-1.66, $\mathrm{p}=0.010$ ), lower compliance (OR 2.80, 95\% CI 1.09$4.74, \mathrm{p}<0.0001$ ), early ambulation (OR 0.92, 95\% CI $0.40-0.99, \mathrm{p}=0.018$ ), stick to discharge criteria (OR 0.89, 95\% CI 0.71-0.97, $\mathrm{p}=0.040$ ) (Table 5).
Table 4 Patients' Clinical Outcomes

\begin{tabular}{|l|l|l|l|}
\hline & $\begin{array}{l}\text { Higher } \\
\text { Compliance } \\
\mathbf{( > 9 2 . 9 )}\end{array}$ & $\begin{array}{l}\text { Lower } \\
\text { Compliance } \\
\mathbf{( \leq 9 2 . 9 )}\end{array}$ & P value \\
\hline LOS, day & $11.8 \pm 4.5$ & $14.6 \pm 6.1$ & $<0.0001$ \\
\hline Any complications & & & 0.031 \\
$\quad$ Yes & 8 & 32 & \\
No & 83 & 137 & 0.355 \\
\hline SSI & 3 & 10 & 0.951 \\
Neurological deficit & 1 & 2 & 0.139 \\
Electrolyte abnormality & 0 & 4 & 0.673 \\
Pneumonia & 1 & 3 & 0.462 \\
DVT/thrombophlebitis & 0 & 1 & 0.462 \\
Pulmonary embolism & 0 & 1 & 0.951 \\
Myocardial infarction & 1 & 2 & 0.298 \\
Urinary tract & 0 & 2 & 0.462 \\
infection & & 1 & 0.462 \\
Stroke & 0 & 1 & 0.718 \\
Sepsis & 0 & 5 & 0.298 \\
Delirium & 2 & 2 & \\
30-day readmission & 0 & & \\
\hline
\end{tabular}


Table 5 Logistic Regression for Prolonged LOS

\begin{tabular}{|c|c|c|c|c|}
\hline \multirow[t]{2}{*}{ Characteristics } & \multicolumn{2}{|c|}{ Univariable Analysis } & \multicolumn{2}{|c|}{ Multivariable Analysis } \\
\hline & OR $(95 \% \mathrm{Cl})$ & p value & OR $(95 \% \mathrm{Cl})$ & p value \\
\hline Age & $1.13(1.01-1.34)$ & 0.022 & $1.45(1.02-1.66)$ & 0.010 \\
\hline Gender (Female) & $0.92(0.66-2.03)$ & 0.764 & & \\
\hline BMI & $0.96(0.91-1.03)$ & 0.893 & & \\
\hline Surgical level $\geq 3$ & $1.35(1.08-2.79)$ & 0.021 & $1.23(0.77-2.55)$ & 0.087 \\
\hline$A S A \geq 3$ & $1.02(0.74-1.87)$ & 0.796 & & \\
\hline Smoker & $1.42(0.77-3.06)$ & $0.84 I$ & & \\
\hline Preoperative ODI (\%) & $1.50(1.02-1.99)$ & 0.045 & $1.62(0.84-2.21)$ & 0.139 \\
\hline Preoperative VAS back & $0.95(0.74-1.08)$ & 0.259 & & \\
\hline Preoperative VAS leg & $1.21(0.94-1.79)$ & 0.632 & & \\
\hline Surgical time & $1.44(1.08-3.76)$ & 0.023 & $1.89(0.95-3.13)$ & 0.228 \\
\hline Estimated blood loss & $1.30(0.8 \mathrm{I}-\mathrm{I} .95)$ & 0.516 & & \\
\hline Lower compliance & $2.98(1.10-4.89)$ & $<0.0001$ & $2.80(1.09-4.74)$ & $<0.0001$ \\
\hline Patient education & $0.97(0.66-3.45)$ & 0.437 & & \\
\hline Nutritional counselling & $1.25(0.83-2.23)$ & 0.278 & & \\
\hline No prolonged fasting & $1.96(0.55-4.46)$ & 0.804 & & \\
\hline Fluid and carbohydrate loading & $0.88(0.24-3.28)$ & 0.633 & & \\
\hline Antithrombotic prophylaxis & $0.90(0.48-2.22)$ & 0.325 & & \\
\hline Antimicrobial prophylaxis & NA & NA & & \\
\hline Tranexamic acid & NA & NA & & \\
\hline Avoidance of salt and water overload & NA & NA & & \\
\hline Maintenance of normothermia & NA & NA & & \\
\hline Local infiltration analgesia & NA & NA & & \\
\hline Early ambulation & $0.83(0.37-0.98)$ & 0.014 & $0.92(0.40-0.99)$ & 0.018 \\
\hline Early removal of bladder catheter & $0.50(0.13-2.12)$ & 0.498 & & \\
\hline Early oral feeding & $0.82(0.59-1.49)$ & 0.153 & & \\
\hline Stick to discharge criteria & $0.94(0.75-0.98)$ & 0.042 & $0.89(0.7 \mathrm{I}-0.97)$ & 0.040 \\
\hline Perioperative multimodal analgesia & NA & NA & & \\
\hline
\end{tabular}

Abbreviations: ASA, American Society of Anesthesiologists; ODI, Oswestry Disability Index; VAS, Visual Analogue Scale.

\section{Discussion}

In this study, the overall compliance of our ERAS program was high, especially for the pre-operative and intra-operative items pre-operative and intra-operative items. Thorn et al considered that compliance of post-operative required more participation of the patients, which is less easily achieved. ${ }^{14}$ Pre-operative and intra-operative items are generally "passive" compliance, where an intervention is delivered to the patient without their direct contribution. ${ }^{14}$ With effective multidisciplinary systems and sufficient education, a high level of passive compliance is easier to achieve.

Our results showed that greater compliance is more easily achieved in patients who are younger. Likewise, Feroci et al also reached the similar conclusion despite variation in types of surgery. ${ }^{15}$ Patients with advanced age are more likely to suffered from cognitive decline, depression, more comorbidities and poor motor activity. ${ }^{16-18}$ These can lead to the delayed recovery in elderly patients, especially those who are frail, unable to ambulate and feed early. Moreover, elderly patients commonly experienced issues of decreased ability to perform daily activities and difficulty with self-care, which leads to reluctance to discharge, even if the criteria are met. ${ }^{19,20}$ Although there was relatively sufficient preoperative education in our study, some elderly patients still had deviations in understanding of the ERAS items and difficulties in early recovery. Therefore, it is necessary to communicate effectively with care-givers and provide elderly patients with various forms of education including handouts preoperatively. Indeed, other studies found no significant variation in compliance with ERAS according to age. ${ }^{21,22}$ Considering that lumbar fusion surgery is performed for patients who present lower motor neuron signs such as radicular symptoms and frailty, which exacerbates the difficulty of complying with ERAS program among elderly patients.

In this study, we showed that high compliance correlated with lower complication rates and reduced rates of prolonged LOS. Similarly, in the setting of other surgeries, it was reported that adverse post-operative outcomes (complications and prolonged 
LOS) were significantly increased with reducing compliance. ${ }^{11,23,24}$ Therefore, it is crucial to maintain compliance in order to improve the prognosis of patients. Iniesta et al suggested that the constant auditing and analysis of the results are helpful to ensure all or most elements of ERAS program are enforced. ${ }^{6}$ What's more, it is easier to maintain compliance when there are strict definitions and standardized elements of the ERAS program.

The lack of research on compliance with individual ERAS elements hindered our understanding of their actual contributions. Our study revealed that the failure of early oral feeding was associated with increased complications. And the failure of early ambulation or sticking to discharge criteria was significantly more influential on prolonged LOS than other elements of the ERAS program. Early feeding can promote the early recovery of gastrointestinal function and reduce risks of electrolyte abnormality. ${ }^{25,26}$ A recent study has proved that early ambulation after elective lumbar spine surgery is associated with significant improvements in LOS, urinary tract infection (UTI) and ileus. ${ }^{27}$ On the other hand, due to factors beyond common discharge criteria, such as logistical and social issues, the delays of elderly patients in discharge directly increase LOS. ${ }^{28}$ The other ERAS items were not independently linked to the prognosis indicators in our study. Nonetheless, the ERAS program is a multimodal pathway and all elements have an additive effect on prognosis. ${ }^{29}$ It can be seen that compliance with the ERAS program is related to the outcomes, but the patients who do not achieve full compliance still benefit from the it.

Our research has some limitations. Our follow-up time was not long enough to evaluate the long-term prognosis and it was just a single-center study with a relatively small sample size. Moreover, we cannot determine whether compliance drives the outcomes or if patients who suffered from a complication were unable to comply with the elements of the ERAS program.

\section{Conclusion}

In summary, we found that low compliance with ERAS program among elderly patients undergoing lumbar fusion surgery was associated with a higher incidence of complications and prolonged LOS. Moreover, some individual elements of the pathway were more effective than others in reducing postoperative complications and LOS. The failure of early oral feeding was associated with increased complications and the failure of early ambulation or sticking to discharge criteria was significantly more influential on prolonged LOS.

\section{Data Sharing Statement}

Request for data sets generated and analyzed during the current study can be addressed to the corresponding author.

\section{Ethics Approval and Consent to Participate}

This study was reviewed and approved by the institutional review board following the declaration of Helsinki principles in Xuanwu Hospital Capital Medical University. The data collection process was entirely anonymous. The types of questions asked could not bring up any singular information that would make participants identifiable in the database and the results are presented in an aggregate manner. A written informed consent was obtained from all participants of this study.

\section{Acknowledgments}

We thank the staff at the Department of Orthopedics, Xuanwu Hospital Capital Medical University, and all the patients who participated in the study.

\section{Author Contributions}

ZEL was responsible for designing the search strategy, evaluating the articles, running statistical analysis and writing this article. CK was responsible for study execution, English editing and substantially revising this article. SBL was responsible for designing the protocol and substantially revising this article. WZS was responsible for drafting the article, interpreting and analyzing results. PW and STZ were responsible for acquisition of data, formatting the article and substantially revising this article. All authors made a significant contribution to the work reported, whether that is in the conception, study design, execution, acquisition of data, analysis and interpretation, or in all these areas; took part in drafting, revising or critically reviewing the article; gave final approval of the version to be published; have agreed on the journal to which the article has been submitted; and agree to be accountable for all aspects of the work.

\section{Funding}

This study was sponsored by National Natural Science Foundation of China [No. 81672201 and No. 81871794]. The funders played no role in the design of this study, the collection, analysis and interpretation of data or preparation of the manuscript.

\section{Disclosure}

The authors declare that they have no competing interests.

\section{References}

1. Kehlet H. Multimodal approach to control postoperative pathophysiology and rehabilitation. Br J Anaesth. 1997;78(5):606-617. doi:10.1093/bja/78.5.606 
2. Wilmore DW, Kehlet H. Management of patients in fast track surgery. $\mathrm{Br}$ Med J. 2001;322(7284):473-476. doi:10.1136/bmj.322.7284.473

3. Brusko GD, Kolcun JPG, Heger JA, et al. Reductions in length of stay, narcotics use, and pain following implementation of an enhanced recovery after surgery program for 1- to 3-level lumbar fusion surgery. Neurosurg Focus. 2019;46(4):1-6. doi:10.3171/2019.1.FOCUS18692

4. Lamperti M, Tufegdzic B, Avitsian R. Management of complex spine surgery. Curr Opin Anaesthesiol. 2017;30(5):551-556. doi:10.1097/ ACO.0000000000000494

5. Puvanesarajah V, Nourbakhsh A, Hassanzadeh H, Shimer AL, Shen FH, Singla A. Readmission rates, reasons, and risk factors in elderly patients treated with lumbar fusion for degenerative pathology. Spine. 2016;41 (24):1933-1938. doi:10.1097/BRS.0000000000001631

6. Iniesta MD, Lasala J, Mena G, et al. Impact of compliance with an enhanced recovery after surgery pathway on patient outcomes in open gynecologic surgery. Int J Gynecol Cancer. 2019;29(9):1417-1424. doi:10.1136/ijgc-2019-000622

7. Durrand JW, Batterham AM, Danjoux GR. Pre-habilitation (i): aggregation of marginal gains. Anaesthesia. 2014;69(5):403-406. doi:10. 1111/anae. 12666

8. Ljungqvist $\mathrm{O}$, Hubner M. Enhanced recovery after surgery-ERAS - principles, practice and feasibility in the elderly. Aging Clin Exp Res. 2018;30(3):249-252. doi:10.1007/s40520-018-0905-1

9. Pisarska M, Torbicz G, Gajewska N, et al. Compliance with the ERAS protocol and 3-year survival after laparoscopic surgery for non-metastatic colorectal cancer. World $J$ Surg. 2019;43 (10):2552-2560. doi:10.1007/s00268-019-05073-0

10. Bagnall NM, Malietzis G, Kennedy RH, Athanasiou T, Faiz O, Darzi A. A systematic review of enhanced recovery care after colorectal surgery in elderly patients. Color Dis. 2014;16(12):947-956. doi:10.1111/codi. 12718

11. Gustafsson UO, Hausel J, Thorell A, Ljungqvist O, Soop M, Nygren J. Adherence to the enhanced recovery after surgery protocol and outcomes after colorectal cancer surgery. Arch Surg. 2011;146 (5):571-577. doi:10.1001/archsurg.2010.309

12. Arrick L, Mayson K, Hong T, Warnock G. Enhanced recovery after surgery in colorectal surgery: impact of protocol adherence on patient outcomes. J Clin Anesth. 2019;55:7-12. doi:10.1016/j.jclinane.2018. 12.034

13. Wang P, Wang Q, Kong C, et al. Enhanced recovery after surgery (ERAS) program for elderly patients with short-level lumbar fusion. J Orthop Surg Res. 2020. doi:10.1186/s13018-020-01814-3

14. Thorn C, White I, Malietzis G, Burch J, Kennedy R, Jenkins JT. "Active compliance" within an ERAS protocol: altering perspective to predict outcome. Color Dis. 2013;15:32. doi:10.1111/codi.12343

15. Feroci F, Lenzi E, Baraghini M, et al. Fast-track surgery in real life: how patient factors influence outcomes and compliance with an enhanced recovery clinical pathway after colorectal surgery. Surg Laparosc Endosc Percutaneous Tech. 2013;23(3):259-265. doi:10. 1097/SLE.0b013e31828ba16f

16. Hsieh TJ, Chang HY, Wu IC, et al. Independent association between subjective cognitive decline and frailty in the elderly. PLoS One. 2018;13(8):e0201351. doi:10.1371/journal.pone.0201351
17. Collard RM, Arts MHL, Schene AH, Naarding P, Oude Voshaar RC, Comijs HC. The impact of frailty on depressive disorder in later life: findings from the Netherlands Study of depression in older persons. Eur Psychiatry. 2017;43:66-72. doi:10.1016/j.eurpsy.2017.01.003

18. Liang YD, Zhang YN, Li YM, et al. Identification of frailty and its risk factors in elderly hospitalized patients from different wards: a cross-sectional study in China. Clin Interv Aging. 2019;14: 2249-2259. doi:10.2147/CIA.S225149

19. Røsstad T, Salvesen Ø, Steinsbekk A, Grimsmo A, Sletvold O, Garåsen $\mathrm{H}$. Generic care pathway for elderly patients in need of home care services after discharge from hospital: a cluster randomised controlled trial. BMC Health Serv Res. 2017;17(1). doi:10.1186/s12913-017-2206-3

20. Nielsen LM, Gregersen Østergaard L, Maribo T, Kirkegaard H, Petersen KS. Returning to everyday life after discharge from a shortstay unit at the emergency department - a qualitative study of elderly patients' experiences. Int J Qual Stud Health Well-Being. 2019;14 (1):1563428. doi:10.1080/17482631.2018.1563428

21. Shiono S, Endo M, Suzuki K, Hayasaka K. Impact of enhanced recovery after surgery on outcomes of elderly patients undergoing open thoracic surgery. Gen Thorac Cardiovasc Surg. 2019;67 (10):867-875. doi:10.1007/s11748-019-01099-2

22. Lohsiriwat V. Outcome of enhanced recovery after surgery (ERAS) for colorectal surgery in early elderly and late elderly patients. Ann Acad Med Singapore. 2019;48(11):347-353.

23. Forster C, Doucet V, Perentes JY, et al. Impact of compliance with components of an ERAS pathway on the outcomes of anatomic VATS pulmonary resections. J Cardiothorac Vasc Anesth. 2020;34 (7):1858-1866. doi:10.1053/j.jvca.2020.01.038

24. Wijk L, Udumyan R, Pache B, et al. International validation of enhanced recovery after surgery society guidelines on enhanced recovery for gynecologic surgery. Am J Obstet Gynecol. 2019;221 (3):237.e1-237.e11. doi:10.1016/j.ajog.2019.04.028

25. Jochum SB, Ritz EM, Bhama AR, Hayden DM, Saclarides TJ, Favuzza J. Early feeding in colorectal surgery patients: safe and cost effective. Int J Colorectal Dis. 2020;35(3):465-469. doi:10.10 07/s00384-019-03500-1

26. Wang J, Yang M, Wang Q, Ji G. Comparison of early oral feeding with traditional oral feeding after total gastrectomy for gastric cancer: a propensity score matching analysis. Front Oncol. 2019;9. doi:10.3389/fonc.2019.01194.

27. Zakaria HM, Bazydlo M, Schultz L, et al. Ambulation on postoperative day \#0 is associated with decreased morbidity and adverse events after elective lumbar spine surgery: analysis from the michigan spine surgery improvement collaborative (MSSIC). Neurosurgery. 2020;87(2):320-328. doi:10.1093/neuros/nyz501

28. Rumstadt B, Guenther N, Wendling P, et al. Multimodal perioperative rehabilitation for colonic surgery in the elderly. World J Surg. 2009;33(8):1757-1763. doi:10.1007/s00268-009-0018-2

29. Kehlet H. Fast-track surgery - an update on physiological care principles to enhance recovery. Langenbeck's Arch Surg. 2011;396 (5):585-590. doi:10.1007/s00423-011-0790-y
Clinical Interventions in Aging

\section{Publish your work in this journal}

Clinical Interventions in Aging is an international, peer-reviewed journal focusing on evidence-based reports on the value or lack thereof of treatments intended to prevent or delay the onset of maladaptive correlates of aging in human beings. This journal is indexed on PubMed Central, MedLine, CAS, Scopus and the Elsevier
Bibliographic databases. The manuscript management system is completely online and includes a very quick and fair peer-review system, which is all easy to use. Visit http://www.dovepress.com/ testimonials.php to read real quotes from published authors. 